\title{
TUBERCULOSE PULMONAR: ASSOCIAÇÃO ENTRE EXTENSÃO DE LESÃO PULMONAR RESIDUAL E ALTERAÇÃO DA FUNÇÃO PULMONAR
}

Rita de Cássia Santa Cruz*, Maria de Fátima Pessoa Militão de Albuquerque, Antônio Roberto leite Campelo, Eduardo Just da Costa e Silva, Edmílson Mazza, Renata Carneiro Menezes, Samuel Kosminsky

Trabalho realizado no Hospital das Clínicas da Universidade Federal de Pernambuco - UFPE - Pós-Graduação em Medicina Tropical - Departamento de Medicina Clínica da UFPE, Recife, PE

*Correspondência:

Rua Paraíso do Norte, 150,

$102-B$

Várzea - CEP 50740-260 -

Recife-PE

Tele/Fax: (81) 34161272

ritascruz@ig.com.br

\begin{abstract}
RESUMO
OвJEtıvo. Descrever as alterações nas variáveis da espirometria e seus distúrbios; estudar a existência de associação entre a extensão da lesão pulmonar residual ao final do tratamento da tuberculose e a alteração da função pulmonar aferida pela espirometria.

Métodos. Estudou-se uma série de 96 pacientes com diagnóstico de tuberculose pulmonar, atendidos em três unidades de saúde de Região Metropolitana do Recife, durante o período de janeiro de 2003 a novembro de 2005. Foram analisados pacientes de ambos os sexos, com idade a partir de 15 anos, cujas radiografias do tórax do final do tratamento foram classificadas pelo critério da National Tuberculosis Association (NTA). Os pacientes responderam questionário, no início da pesquisa, e foram submetidos à prova de função pulmonar, após o término do tratamento.

Resultados. Dos 96 pacientes estudados, conclúmos que 89,6\% apresentavam seqüelas radiográficas; 54\% apresentavam seqüelas moderadas a graves. Estas alterações radiográficas correspondiam a $24,6 \%$ e $73,8 \%$, respectivamente, de alterações na função pulmonar.

ConcLusäo. Foram identificadas $89,6 \%$ de lesões residuais radiográícas, enquanto $66,7 \%$ das disfunções respiratórias alertam para o fato de que a assistência ao paciente com tuberculose pulmonar não deve se restringir apenas à cura baciloscópica da doença. A identificação de lesões pulmonares residuais e disfunções respiratórías em pacientes que concluem o tratamento da tuberculose permitirão instituir medidas precoces de tratamento dessas seqüelas para uma melhor qualidade de vida.
\end{abstract}

UnITERMOS: Tuberculose pulmonar. Espirometria. Disfunção respiratória.

\section{INTRODUÇÃo}

A importância da detecção precoce dos casos novos de tuberculose pulmonar (TB) decorre das evidências encontradas em alguns estudos em que o período de tempo, decorrido entre o início de sintomas de um paciente com tuberculose pulmonar até a instituição do tratamento, contribui para uma maior disseminação da doença e para a progressão das lesões pulmonares ${ }^{1-4}$.

A presença de lesões pulmonares residuais extensas pode ser um fator preditor de invalidez permanente por conta de insuficiência respiratória secundária à destruição tecidual, cor pulmonale e predisposição a infecções oportunísticas, com prejuízo para a qualidade de vida². Além disso, extensão da doença é um dos fatores de riscos implicados na mortalidade por $\mathrm{TB}^{5}$.

Apesar da radiografia do tórax não ser o método recomendado pelo Ministério da Saúde, para acompanhar a resposta ao tratamento por conta da imprecisão diagnóstica, é um exame capaz de determinar o grau de acometimento pulmonar secundário à tuberculose, podendo ser solicitado em unidades primárias de saúde. Além disso, a radiografia do tórax pode contribuir para a comparação com exame prévio e avaliação final do tratamento. Outros trabalhos mostram vantagem da tomografia do tórax para avaliar as lesões pulmonares sendo, porém, um método dispendioso e não acessível na maioria das unidades de saúde que prestam assistência ao doente com TB pulmonar ${ }^{6,7}$.

Por não haver dados na literatura nacional sobre a existência de associação entre presença de seqüela à radiografia do tórax e a alteração da função pulmonar aferida pela espirometria ${ }^{8}$, realizamos o presente trabalho. Procuramos descrever as alterações nas variáveis da espirometria e seus distúrbios; estudar a existência de associação entre a extensão da lesão pulmonar residual ao final do tratamento da tuberculose e a alteração da função pulmonar aferida pela espirometria.

\section{Métodos}

A população do estudo é de 96 pacientes de ambos os sexos, residentes na Região Metropolitana de Recife, diagnosticados e tratados em três unidades de saúde: Hospital das Clínicas da UFPE; Policlínica, da Prefeitura de Recife; e Policlínica, da Prefeitura de Jaboatão dos Guararapes, referências para atendimento da tuberculose, no período de janeiro de 2003 a agosto de 2005.

- Critérios de inclusão: Todos os pacientes com idade a partir de 15 anos que procuraram as unidades de saúde selecionadas, acometidos de tuberculose pulmonar, e possuíam radiografia de 
tórax no início do tratamento. Realizaram o tratamento nestes serviços, e após alta por cura, se submeteram a uma radiografia do tórax e prova de função pulmonar.

- Critérios de exclusão: Mulheres com atraso menstrual ou aquelas que sabiam estar grávida, pelo risco da radiação; pacientes soropositivo para HIV.

Desenho do Estudo: Série de casos de tuberculose pulmonar tratada com grupos de comparação com e sem disfunção respiratória após o tratamento.

\section{Definição das variáveis}

- Variável dependente: disfunção pulmonar pós-tratamento da tuberculose pulmonar.

Segundo as Diretrizes para Testes de Função Pulmonar?a a espirometria foi classificada como normal quando a capacidade vital forçada (CVF), o volume expiratório forçado no primeiro segundo $\left(V_{E F}\right.$ ) e o Tiffenau (VEF, / CVF) eram iguais ou superiores a $80 \%$ do previsto; restritiva, quando a capacidade vital forçada (CVF) estava abaixo de $80 \%$ do previsto; e obstrutiva, quando o volume expiratório forçado em I segundo (VEF, foi menor que $80 \%$ do previsto. Quando o índice de Tiffenau (VEF,/CVF) estava abaixo de $80 \%$ foi classificada como obstrutiva. A prova de função pulmonar foi, sempre, realizada no final do tratamento.

Consideramos, neste estudo, que existe disfunção (seqüela) pulmonar na presença de alteração de quaisquer dos parâmetros acima.

\section{- Variáveis independentes - características dos casos:}

I. Definição de seqüela na radiografia do tórax.

Conceitua-se seqüela radiográfica da lesão pulmonar, por tuberculose, reação fibrótica no pulmão, cuja extensão varia de lesão mínima à avançada ${ }^{\prime \prime, 12}$. A extensão após o tratamento foi mensurada mediante adaptação de critério simples da National Tuberculosis Association (NTA), utilizada para categorização da extensão da lesão pulmonar inicial da tuberculose ${ }^{8}$.

Para a classificação das seqüelas utilizamos classificação do NTA, porém os tipos de lesões deixaram de apresentar os infiltrados e cavidades de paredes espessas, por serem características de atividade do processo, para se tornarem alterações residuais: bandas parenquimatosas, bronquiectasias, fibrose, cavidade de paredes finas.

- NTA I ou mínima - lesão pode atingir pequena parte de um ou ambos os pulmões, mas suas extensões totais, independentes da distribuição, não devem exceder o volume do pulmão, correspondente ao que está acima da segunda articulação condroesternal, e espinhal da quarta articulação ou corpo da quinta vértebra dorsal de um só lado, ou seja, atingir até o terço superior de um dos pulmões;

- NTA II ou moderadamente avançada - lesão pode estar em um ou em ambos os pulmões. Sua extensão não deve exceder o volume correspondente a todo um pulmão se as lesões não são confluentes. Se as lesões são confluentes, não devem ocupar mais que o equivalente a um terço do pulmão;

- NTA III ou muito avançada - aquela que ultrapassa o limite da moderada.

2. Sexo;

3. Idade: 15-39 anos; 40-59 anos; $\geq 60$ anos;

\begin{tabular}{lccc}
\hline \multicolumn{4}{c}{ Tabela I - Quantificação dos distúrbios da função pulmonar ${ }^{10}$} \\
\hline Distúrbio & VEF $_{1}$ & CVF & VEF/CVF \\
\hline Leve & $60 \%-L I$ & $60 \%-L I$ & $60 \%-L \mid$ \\
Moderado & $41 \%-59 \%$ & $51 \%-59 \%$ & $41 \%-59 \%$ \\
Grave & $\leq 40 \%$ & $\leq 50 \%$ & $\leq 40 \%$ \\
\hline
\end{tabular}

4. Tabagismo: Hábito de fumar cigarros. Os pacientes foram categorizados como fumantes, não-fumantes, e ex-fumantes:

a) Considerou-se fumante o consumidor de, pelo menos, um cigarro diário, por período não inferior a um mês, ou aquele que cessou o hábito de fumar, havia menos de 12 meses.

b) Ex-fumante é todo aquele que se encontra, há 12 meses, sem fumar, de acordo com o Manual sobre Tabagismo da SBPT ${ }^{13}$.

Os fumantes para participarem do estudo eram previamente hígidos.

5. Tempo de doença: definido como o período de tempo desde o início dos sintomas até o início do tratamento e categorizado em dias: até 30 dias, 30-60 dias e acima de 60 dias;

6. Comorbidades (morbidade auto-referida): relato da presença / ausência de doenças que levem à imunodepressão, através de diagnóstico feito por médico ${ }^{14,15}$. Os pacientes com comorbidades eram todos hígidos.

7. Etilismo: Os pacientes foram categorizados em etilistas com baixo risco ou alto risco, de acordo com a quantidade de álcool ingerido, baseado na fórmula usada pelo programa Viva Mais da UNICAMP':

Baixo Risco - Mulheres quando ingerem menos de 7 latas de cerveja, por semana; e homens, menos de 14 latas de cerveja, por semana;

Alto Risco - Mulheres que ingerem de $\geq 7$ latas de cerveja, por semana, e homens, $\geq 14$ latas de cerveja, por semana.

Uma lata de cerveja $=17 \mathrm{~g}$ de álcool $=$ uma taça pequena de vinho $(\mid 40 \mathrm{ml})=$ uma dose de MartiniNermute $(50 \mathrm{ml})=$ uma dose de aguardente ou vodca ou uísque $(37 \mathrm{ml})$.

\section{Coleta de dados}

Os pacientes incluídos no estudo foram entrevistados para o preenchimento de questionários. Apenas um radiologista analisou as radiografias no final do tratamento em dois momentos distintos.

A espirometria para identificar a presença de seqüelas funcionais foi realizada no laboratório de função pulmonar do Serviço de Pneumologia do Hospital das Clínicas por um mesmo técnico que desconhecia o quadro dos pacientes. O espirômetro foi o Beatrice AT, da EBEM, que tem como padrão de gerenciamento o Pulmosoft, que oferece opção de uso do padrão brasileiro para cálculo do valor teórico de adultos, o padrão Pereira?.

\section{Análise estatística}

Para análise dos dados foram obtidas distribuições absolutas e relativas uni e bivariadas e as medidas estatísticas: média, mediana e desvio padrão da variável, idade e utilizado os teste Qui quadrado de Pearson ou o teste Exato de Fisher, quando as condições para utilização 
do teste Qui quadrado não foram verificadas. Os resultados também incluíram os valores da razão de prevalência (RP) e intervalos de confiança (IC) para esses parâmetros ${ }^{17,18}$,

Os dados foram digitados em Excel; o programa utilizado para obtenção dos cálculos estatísticos foi o Statistical Analysis Sistem (SAS) na versão 8. O nível de significância utilizado para a obtenção dos cálculos estatísticos foi de $5 \%$.

\section{Resultados}

A idade mínima dos 96 pacientes foi 15 e a máxima foi de 72 anos, com média de 4I,08 anos, desvio padrão de I4,32 anos e mediana de 41,50 anos.

Foi observado que $54,2 \%$ dos pacientes eram do sexo masculino. As duas faixas etárias mais prevalentes foram: 15 a 39 anos e 40 a 59 anos, representando $45,8 \%$ e $42,7 \%$ do total da amostra, respectivamente. Mais da metade (58,3\%) dos pacientes tinha mais de 60 dias da doença e o menor percentual (14,6\%) correspondeu aos que tinham a doença até 30 dias; cerca de 38,5\% eram tabagistas e 19,6\%, eram etilistas, com a ressalva de que apenas $2 \%$ bebiam uma quantidade grande (número de latas de cerveja $\geq 14$, no caso dos homens, e $\geq 7$, no caso das mulheres). A presença de co-morbidades foi registrada em |4,6\% da amostra. A extensão da lesão foi do tipo NTA III, para a maioria (61,5\%) dos pacientes, seguida de $30,2 \%$, que tinham NTA Il; e para os 8,3\% dos pacientes restantes, a extensão da lesão foi considerada NTA I.

A distribuição dos pacientes pesquisados, segundo as variáveis da espirometria, mostrou que $37,5 \%$, tinham o CVF normal, sendo que o maior percentual (47,9\%) correspondeu aos que tinham o CVF reduzido no grau leve; 10,4\% apresentavam redução moderada e 4,2\% eram graves; $40,6 \%$ tinham o VEF, normal e 33,3\% tinham o VEF, diminuído no grau leve; a maioria (85,4\%) tinha o Tiffenau normal, $13,5 \%$ tinham redução leve e nenhum apresentou o Tiffenau no grau grave.

$\mathrm{Na}$ Tabela 2 analisamos o tipo e grau de disfunção respiratória.

Quando analisamos disfunção respiratória e extensão da lesão pulmonar residual (Tabela 3), verificamos associação no NTA pósnormal em $20 \%$ dos casos, NTA pós I em 53, $1 \%$ dos casos; NTA pós II em 81,1\% dos casos; NTA pós III em 71,4\% dos casos. Para calcularmos a razão de prevalência (RP), agregamos como grau I o NTA pós- normal e I, e como grau 2 o NTA pós II e III. No grau I, 19 casos (45,2\%) tiveram disfunção respiratória e 23 (54,8\%) não tiveram disfunção respiratória. No grau 2, 45 (83,3\%) tiveram disfunção respiratória e nove (16,7\%) não tiveram disfunção respiratória.

Verificamos que existe uma associação entre o grau de extensão da lesão pulmonar residual e disfunção respiratória com o risco de 1,8 vezes, mostrando-se estatisticamente significante ao nível de 5\% $(p<0,05)$.

\section{Discussão}

Existem poucos estudos avaliando as conseqüências da TB pulmonar após o tratamento, demonstrável pela radiografia do tórax, e que possa inferir algum distúrbio na função pulmonar. $\mathrm{O}$ tratamento da tuberculose pode conduzir à melhora na função pulmonar, segundo Plit et al. ${ }^{19}$, que consideravam o escore radiográfico pré e pós tratamento

\begin{tabular}{lcc}
\hline \multicolumn{2}{c}{$\begin{array}{c}\text { Tabela 2 - Distribuição dos pesquisados, segundo o tipo de alteração } \\
\text { respiratória (restritivo ou obstrutivo) }\end{array}$} \\
\hline Condição grespirau de disfunçária & N & 2003-2005 \\
\hline - Normal & 32 & 33,3 \\
- Restritivo & 38 & 39,6 \\
Restritivaleve \\
Restritiva moderada \\
Restritiva grave \\
- Obstrutivo & 2 & 2,1 \\
Obstrutivaleve & - & - \\
Obstrutivamoderada & 3 & \\
Obstrutivagrave & 7 & 3,1 \\
- Mista - restritivo e obstrutivo & 2 & 7,3 \\
Total & 12 & 2,1 \\
\hline & 96 & 12,5 \\
& & 100,0 \\
\hline
\end{tabular}

como marcador da extensão do envolvimento da TB no parênquima pulmonar.

A nossa casuística reflete a situação epidemiológica da tuberculose no nosso meio: dos 15 aos 59 anos, concentram-se $88,5 \%$ da amostra, com uma baixa prevalência em idosos, apenas I I casos; o predomínio discreto no sexo masculino, representando 54,2 \% da amostra; um marcante retardo no diagnóstico e no início do tratamento, com 27, $1 \%$ da amostra com diagnóstico após 30 dias e 58,3\% após 60 dias de doença.

Retardo no diagnóstico e no início do tratamento é um dos fatores que são implicados na literatura em uma maior lesão no parênquima pulmonar e, conseqüentemente, na sua função pulmonar ${ }^{6,7,11}$. Corroborando esse pensamento, em 61,5\% dos nossos casos, a radiografia inicial foi classificada como NTA III, e o diagnóstico em 58,3\% dos pacientes foi realizado com mais de 60 dias de doença. No nosso estudo verificamos que mais da metade dos casos $(58,3 \%)$ tinha mais de 60 dias de doença, quando do iníciodo tratamento. Altet-Gómez et al. ${ }^{20}$ consideram que 0 atraso não deve exceder 30 dias porque as alterações estruturais do parênquima são mais intensas e cuja reversão torna-se mais difícil.

Após o tratamento, foi observado que o grau de extensão da lesão pulmonar residual, categorizada como grau I (leve) e grau 2 (grave) teve associação com disfunção respiratória, estatisticamente significante, com um risco de I,8 vezes desenvolver essa disfunção. No grau I, tivemos 19 casos (45,2\%) com disfunção respiratória e 23 casos $(54,8 \%)$ sem disfunção respiratória. E no grau 2 tivemos 45 casos $(83,3 \%)$ com disfunção respiratória e nove casos (16,7\%) sem disfunção respiratória. Campos et al. ${ }^{21}$, utilizando a mesma análise do NTA, observaram em 29 pacientes que apresentavam extensão da lesão residual NTAll (6/29) ou III (23/29). Ramos et al. ${ }^{22}$ encontraram $38 \%$ dos pacientes com NTA I, 35\% com NTA II e 27\% com NTA III, e que quanto mais acentuada a lesão, maior a intensidade do distúrbio, sendo estatisticamente significante.

No nosso estudo foram encontrados em $66,7 \%$ da amostradistúrbios ventilatórios. Predominam restrição leve em 39,6\% (38/96) e distúrbio misto em 12,5\% (12/96). Lee e Chung ${ }^{23}$, ao estudarem, estenose endobrônquica secundária a TB, a função 
Tabela 3 - Avaliação da disfunção respiratória segundo cada uma das variáveis: sexo, faixa etária, tempo de doença, tabagismo, etilismo, outras doenças e grau da extensão residual pulmonar (NTA pós), 2003-2005.

\begin{tabular}{|c|c|c|c|c|c|c|c|c|c|c|c|}
\hline \multirow{3}{*}{ Variável } & & \multicolumn{5}{|c|}{ Disfunção respiratória } & & \multirow{2}{*}{\multicolumn{2}{|c|}{ TOTAL }} & \multirow{3}{*}{ Valor de $p$} & \multirow{3}{*}{ RP (IC 95\%) } \\
\hline & & \multicolumn{2}{|c|}{ Sim } & & \multicolumn{2}{|c|}{ Não } & & & & & \\
\hline & & $n$ & $\%$ & & $n$ & $\%$ & & $\mathbf{N}$ & $\%$ & & \\
\hline $\begin{array}{l}\text { - NTA pós } \\
\text { Graul } \\
\text { Graull } \\
\text { Grupototal }\end{array}$ & $\begin{array}{l}19 \\
45 \\
64\end{array}$ & $\begin{array}{l}45,2 \\
83,3 \\
66,7\end{array}$ & $\begin{array}{c}23 \\
9 \\
32\end{array}$ & $\begin{array}{l}54,8 \\
16,7 \\
33,3\end{array}$ & & & $\begin{array}{l}100,0 \\
100,0 \\
100,0\end{array}$ & & & & $\begin{array}{c}1,00 \\
1,84(1,29 a 2,62\end{array}$ \\
\hline $\begin{array}{l}\text { - Sexo } \\
\text { Masculino } \\
\text { Feminino } \\
\text { Grupototal }\end{array}$ & & $\begin{array}{l}35 \\
29 \\
64\end{array}$ & $\begin{array}{l}67,3 \\
65,9 \\
66,7\end{array}$ & & $\begin{array}{l}17 \\
15 \\
32\end{array}$ & $\begin{array}{l}32,7 \\
34,1 \\
33,3\end{array}$ & & $\begin{array}{l}52 \\
44 \\
96\end{array}$ & $\begin{array}{l}100,0 \\
100,0 \\
100,0\end{array}$ & $p^{(l)}=0,8848$ & $\begin{array}{c}1,02(0,46 \text { a } 2,49 \\
1,00\end{array}$ \\
\hline $\begin{array}{l}\text { - Faixa etária } \\
\text { I5 a } 39 \\
40 \text { a } 59 \\
\text { Grupo total }\end{array}$ & & $\begin{array}{l}29 \\
35 \\
64\end{array}$ & $\begin{array}{l}65,9 \\
67,3 \\
66,7\end{array}$ & & $\begin{array}{l}15 \\
17 \\
32\end{array}$ & $\begin{array}{l}34,1 \\
32,7 \\
33,3\end{array}$ & & $\begin{array}{l}44 \\
52 \\
96\end{array}$ & $\begin{array}{l}100,0 \\
100,0 \\
100,0\end{array}$ & $p^{(l)}=0,8848$ & $\begin{array}{c}1,00 \\
1,02(0,77 a 1,36\end{array}$ \\
\hline $\begin{array}{l}\text { - Tempo de da } \\
\text { Até } 30 \text { dias } \\
\text { Mais de } 30 \text { dias }\end{array}$ & & $\begin{array}{c}6 \\
58\end{array}$ & $\begin{array}{l}42,9 \\
70,7\end{array}$ & & $\begin{array}{c}8 \\
24\end{array}$ & $\begin{array}{l}57,1 \\
29,3\end{array}$ & & $\begin{array}{l}14 \\
82\end{array}$ & $\begin{array}{l}100,0 \\
100,0\end{array}$ & $p^{(2)}=0,0634$ & $\begin{array}{c}1,00 \\
1,65(0,89 \text { a } 3,07\end{array}$ \\
\hline Grupototal & & 64 & 66,7 & & 32 & 33,3 & & 96 & 100,0 & & \\
\hline $\begin{array}{l}\text { - Tabagismo } \\
\text { Sim } \\
\text { Não } \\
\text { Grupo total }\end{array}$ & & $\begin{array}{l}29 \\
35 \\
64\end{array}$ & $\begin{array}{l}78,4 \\
59,3 \\
66,7\end{array}$ & & $\begin{array}{c}8 \\
24 \\
32\end{array}$ & $\begin{array}{l}21,6 \\
40,7 \\
33,3\end{array}$ & & $\begin{array}{l}37 \\
59 \\
96\end{array}$ & $\begin{array}{l}100,0 \\
100,0 \\
100,0\end{array}$ & $p^{(1)}=0,0539$ & $\begin{array}{c}1,32(1,01 \text { a } 1,73 \\
1,00\end{array}$ \\
\hline $\begin{array}{l}\text { • Etilismo } \\
\text { Sim } \\
\text { Não } \\
\text { Grupo total }\end{array}$ & & $\begin{array}{l}11 \\
49 \\
60\end{array}$ & $\begin{array}{l}61,1 \\
66,2 \\
65,2\end{array}$ & & $\begin{array}{c}7 \\
25 \\
32\end{array}$ & $\begin{array}{l}38,9 \\
33,8 \\
34,8\end{array}$ & & $\begin{array}{l}18 \\
74 \\
92\end{array}$ & $\begin{array}{l}100,0 \\
100,0 \\
100,0\end{array}$ & $p^{(1)}=0,6834$ & $\begin{array}{c}1,00 \\
1,08(0,72 a 1,62\end{array}$ \\
\hline $\begin{array}{l}\text { - Comorbidad } \\
\text { Sim } \\
\text { Não }\end{array}$ & & $\begin{array}{c}8 \\
56\end{array}$ & $\begin{array}{l}57,1 \\
68,3\end{array}$ & & $\begin{array}{c}6 \\
26\end{array}$ & $\begin{array}{l}42,9 \\
31,7\end{array}$ & & $\begin{array}{l}14 \\
82\end{array}$ & $\begin{array}{l}100,0 \\
100,0\end{array}$ & $p^{(2)}=0,5406$ & $\begin{array}{c}1,00 \\
1,19(0,74 \text { a } 1,92\end{array}$ \\
\hline Grupototal & & 64 & 66,7 & & 32 & 33,3 & & 96 & 100,0 & & \\
\hline
\end{tabular}

$(*)$ - Associação significante a $5 \%$

(1) - Através do teste Qui quadrado de Pearson

(2) - Através do teste Exato de Fisher

pulmonar era normal em 16 casos (23,5\%); restritiva em 32 casos (47\%); obstrutiva em quatro casos (5,9\%); e mista em 14 casos (23,5\%). O CVF e VEF, melhoravam, significantemente, após um mês de tratamento, mas não modificava significantemente entre o primeiro e o sexto meses de tratamento. A melhora do CVF coincidia com os achados broncoscópicos.

Campos et al. ${ }^{21}$ encontraram que o padrão espirométrico predominante era o misto, e essa diferença é, estatisticamente, significante; além disso, o espaço morto foi significantemente maior. Ramos et al. ${ }^{22}$ encontraram distúrbio ventilatório combinado em 34\%; distúrbio obstrutivo em 24\% dos casos; função normal em 24\% dos casos; e distúrbio ventilatório restritivo em $18 \%$ dos casos.

O fato de termos avaliado a radiografia do tórax logo após o tratamento pode superestimar o número de seqüelados porque algumas lesões no parênquima, após o tratamento, podem diminuir ou até desaparecer com o tempo ${ }^{2,24}$. Contrabalançando esse ponto, temos que como não utilizamos tomografia de tórax, porque não constituía nosso objetivo, lesões mínimas podem não ter sido identificadas, inclusive nos 10 pacientes que são considerados sem seqüelas radiográfica ${ }^{25}$.

Apesar do grande número de registro de seqüelas no nosso estudo, o desaparecimento completo das lesões é raro, mas podem ocorrer se a lesão cura antes da necrose se desenvolver ${ }^{8}$.

Este estudo vem contribuir ao alertar que, apesar dos progressos que vêm se verificando no âmbito do diagnóstico da tuberculose, as lesões radiográficas encontradas são, na maior parte das vezes, extensas, raramente são lesões mínimas. Isto fala de diagnóstico tardio e de mais seqüelados na sociedade.

Acreditamos que tratar a tuberculose no início da doença, como forma de curar integralmente o paciente, se torna crucial para o controle da tuberculose e suas seqüelas.

Conflito de interesse: não há 


\section{SUMMARY}

Pulmonary tuberculosis: association between extent of the RESIDUAL PULMONARY LESION AND ALTERATION IN THE LUNG FUNCTION

OBJECTNE. To describe alterations in spirometric variables and its disturbances and to study the existence of a relationship between extent of the residual lung lesion at the end of treatment for tuberculosis and alteration of the lung function measured by spirometry.

METHODS. Analyzed were 96 patients with diagnosis of pulmonary tuberculosis, treated in three health centers in the metropolitan area of Recife, from January, 2003 to November, 2005. Patients of both genders, 15 years of age or older were included, whose chest $x$-rays at end of the treatment were classified according to criteria of the National Tuberculosis Association (NTA) for extent of lung lesion. Patients replied to a questionnaire at the beginning of the survey, and were submitted to spirometry after conclusion of treatment.

RESULTS. Of the 96 patients, $89.6 \%$ presented with radiographic sequels. About 54\% had moderate to severe sequels. These radiographic alterations correspond to $24.6 \%$ and $73.8 \%$ respectively of alterations in the pulmonary function.

CONCLUSION. The large number of residual radiographic lesionss (89.6\%) and breathing dysfunction (66,7\%) identified in this survey call attention to the fact that treatment of a patient with pulmonary tuberculosis must not be restricted to bacteriological healing of the disease. Identification of the residual lung lesions and of the breathing dysfunction in patients who conclude treatment will promote early actions to treat these sequels, improving the quality of life of these patients. [Rev Assoc Med Bras 2008; 54(5): 406-10]

KEY WORDS: Pulmonary tuberculosis. Spirometry. Respiratory dysfunction.

\section{REFERÊNCIAS}

I. Long R, Maycher B, Dhar A, Manfreda J, Hershfield E, Anthonisen N. Pulmonary tuberculosis treated with directly observed therapy- serial changes in lung structure and function. Chest. 1998; I 13:933-42.

2. Kim HY, Song GS, GooJM, Lee JS, Lee KS, Lim TH: Thoracic sequelae and complications of tuberculosis. Radiographics. 200 I;21:839-60.

3. Job JRPP, Gozzano JOA, Bernardes Jr OR, Garcia RH, Miralhes OJC, Miranda MAP. Informações que antecederam o diagnóstico de Tuberculose pulmonar e tempo decorrido até o início do tratamento em pacientes matriculados em centro de saúde, São Paulo (Brasil). Rev Saúde Púbica. 1986;20:21-5.

4. Santos MAPS, Albuquerque MFPM, Ximenes RAA, Lucena-Silva NLCL, Braga C, Campelo ARL, et al. Risk factors for treatment delay in pulmonary tuberculosis in Recife, Brazil. BMC Public Health. 2005;5: I-8.

5. Humphries MJ, Byfield SP, Darbyshire JH, Davies PD, Nunn AJ, et al. Deaths occurring in newly notified patients with pulmonary tuberculosis in England and Wales. Br J Dis Chest. 1984;78: I 49-58.

6. Miranda G, Diaz JC Arancibia P, Antolini M, Diaz C, Vidal A: Manifestaciones radiográficas de la tuberculosis pulmonar. Rev Chil Radiol. 2004; I 0: I 78-82.
7. Jones BE, Ryu R, Yang Z, Cave MD, Pogoda JM, Otaya M, et al. Chest radiographic findings in patients with recent or remote infection. Am J Resp Crit Care Med. 1997; 1 56: I 270-3.

8. Ribeiro SN, Gehardt FG, Silva JRL, Fonseca L, Gontijo P, Sant' Anna CC, et al. Tuberculose. In: Pneumologia. 4a ed. São Paulo: Atheneu; 1995. p.379-448.

9. Sociedade Brasileira de Pneumologia e Tisiologia (SBPT). Diretrizes para testes de função pulmonar. J Pneumol. 2002;28.

10. Silva LCC, Rubin AS, Silva LMC. Avaliação funcional pulmonar. In: Silva LCC, organizador. Condutas em pneumologia. São Paulo: Revinter; 200I.v.l, p. I09-2I.

I I. Winer-Muram HT, Rubin, AS. Thoracic complications of tuberculosis. J Thorac Imaging. 1990;5:46-63.

12. Sociedade Brasileira de Pneumologia e Tisiologia (SBPT). Consenso Brasileiro sobre terminologia dos descritores de tomografia computadorizada do tórax. J Pneumol. 2005;31: | 49-55.

13. Pereira LFF. Manual sobre tabagismo. Sociedade Brasileira de Pneumologia e Tisiologia. [citado 2 I maio 2006]. Disponível em: http:// www.sbpt.org.br.

14. Tattevin P, Casalino E, Fleury L, Egmann G, Ruel M, Bouvet E. The validity of medical history, classic symptoms, and chest radiographs in predicting pulmonary tuberculosis. Chest. 1999; I I : 1 248-53.

15. Biz E, Pereira CAP, Moura LAR, Sesso R, Vaz MLS, Silva Filho AP, et al. The use of cyclosporine modifies the clinical and histopathological presentation of tuberculosis after renal transplantation. Rev Inst Med Trop São Paulo. 2000;42:225-30

16. Viva Mais. Programa de prevenção ao uso de substâncias psicoativas lícitas e ilícitas. São Paulo: UNICAMP; 2006. [citado I 4 maio 2006]. Disponível em: http://www.prdu.unicamp.br/vivamais/.

17. Altman DG. Practical statistics for medical research. London: Chapman \& Hall CRC; 1991. p.611.

18. ZarJH. Biostatistical analysis. $4^{\text {th }}$ ed. New Jersey: Prentice Hall; I 999. p.929.

19. Plit ML, Anderson R, Van Rensburg CE, Page-Shipp L, Blott JA, Fresen JL, et al. Influence of antimicrobial chemotherapy on spirometric parameters and pro-inflammatory indices in severe pulmonary tuberculosis. Eur Respir J. 1998; | 2:35|-6.

20. Altet-Gómez MN, Megías JÁ, Soler JC, Auge CM, Fuentes MAJ, Galvão MLS, et al. Estúdio del retraso diagnóstico de la tuberculosis pulmonar sintomática. Arch Bronconeumol. 2003;39: | 46-52.

21. Campos EP, Campos CEOP, Cataneo AJM. Função pulmonar comparativa da paracoccidioidomicose com a tuberculose. J Pneumol. 1990: 16:62-3.

22. Ramos LMM, Sulmonett N, Ferreira CS, Henriques JF, Miranda SS: Perfil funcional de pacientes portadores de seqüela de tuberculose de um hospital universitário. J Pneumol. 2006;32:43-7.

23. Lee JH, Chung HS. Bronchoscopic, radiologic and pulmonary function evaluation of endobronchial tuberculosis. Respirology. 2000;5:4I I-7.

24. Weg JG. Formas clínicas da doença micobacteriana. In: Fishma AP, editor. Diagnóstico das doenças pulmonares. $2^{a}$ ed. Rio de Janeiro: Manole; 1992. v.3, p. $1895-14$.

25. Im JG, Itoh H, Shim YS, Ahn J, Han MC, Noma S. Pulmonary tuberculosis: CT findings: early active disease and sequential change with antituberculous therapy. Radiology. 1993; 1 86:653-60.

Artigo recebido: 15/07/07

Aceito para publicação: 10/04/08 